$>$ Le bon fonctionnement de la cellule dépend du contrôle fin des mécanismes transcriptionnels et traductionnels. De nombreuses études ont établi que les dérèglements transcriptionnels peuvent être à l'origine de maladies telles que le cancer, l'obésité et le diabète. Depuis une décennie, le rôle de la traduction des ARNm dans certaines de ces pathologies et son contrôle par la voie $\mathrm{PI3K} / \mathrm{Akt} / \mathrm{mTOR}$ a été démontré. La rapamycine, inhibiteur spécifique de mTOR, présente une forte activité anti-proliférative dans plusieurs types de cancer. De récentes études démontrent qu'elle pourrait potentiellement être efficace dans le traitement de l'obésité et du diabète. La rapamycine et ses analogues semblent donc destinés à un avenir prometteur. <

\section{Quand \\ la traduction \\ sort de sa TORpeur}

Olivier Le Bacquer, Yvan Martineau, Yaël Mamane

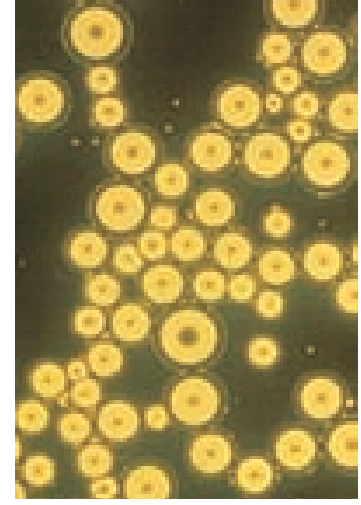

d'elF4B dénaturent les structures secondaires de l'ARNm et facilitent la liaison du ribosome pour finalement déclencher la synthèse protéique (Figure 1) [2]. L'importance physiopathologique de la traduction ne fait aucun doute puisqu'une suractivation traductionnelle peut conduire au cancer, à l'obésité ou au diabète $[3,4]$. Cet article se propose d'approfondir le lien entre la synthèse protéique et ses pathologies.

La traduction, sensible à la disponibilité en nutrimen et en facteurs de croissance, représente une étape clé de l'expression génique impliquée dans la plupart des phases du métabolisme liées à la croissance cellulaire [1]. La synthèse protéique est un processus énergétique très coûteux strictement contrôlé, dès le stade d'initiation de la traduction. Ce contrôle est amorcé grâce à des facteurs d'initiation appelés eukaryotic initiation factors (elF) [2], elF4E étant le plus largement étudié. elF4F permet le positionnement du complexe de pré-initiation 435 à l'extrémité 5' de l'ARN messager (ARNm) (Figure 1), complexe qui analysera l'ARNm pour détecter I'AUG initiateur. Chez les eucaryotes, la traduction de la plupart des ARNm dépend d'une structure en 5' appelée « coiffe » (ou cap) ( $\mathrm{m}^{7} \mathrm{GpppN}$ où N représente n'importe quel nucléotide) et de la présence du complexe elF4F composé de trois sous unités : elF4E, elF4A et elF4G [2]. elF4E est le facteur clé dans la régulation de l'initiation de la traduction: il interagit avec la coiffe, mais aussi avec elF4G (un adaptateur entre les divers facteurs d'initiation) qui se lie à elF4A (une ATPase-hélicase) et elF3 qui associe la sous-unité ribosomique $43 \mathrm{~S}$ pour former le complexe elF4F. Les activités d'elF4A et

Article reçu le 27 juin 2005, accepté le 19 janvier 2006.

\section{Synthèse protéique et cancer}

Les facteurs d'initiation de la traduction

Parmi les facteurs d'initiation de la traduction, elF4E fut le premier impliqué dans la transformation de cellules en culture. Plusieurs ARNm, activement traduits durant la croissance cellulaire et agissant dans la transformation, contiennent des structures secondaires dans leur extrémité 5'non-traduite (vascular endothelial growth factor A, cyclin D1, fibroblast growth factor 2, ornithine decarboxylase) [3]. La surexpression d'elF4E aboutit à un dérèglement de la synthèse protéique et du fonctionnement cellulaire (pour revue, voir [3]) et, en particulier, à une augmentation de la traduction de ces ARNm. D'autres études suggèrent également qu'elF4E serait susceptible de coopérer avec d'autres protéines transformantes telles que c-Myc et $\varepsilon 1 A[3,5,6]$ et que son expression serait particulièrement élevée dans plusieurs types de cancer (pour revue, voir [3]). De façon similaire, le complexe elF4F est nécessaire au maintien de la croissance des cellules cancéreuses mammaires in vivo [7]. Enfin, d'autres facteurs déclencheurs tels 
qu'elF4G, elF4A et elF3 jouent un rôle important dans l'oncogenèse [3]. Ce constat renforce l'idée qu'une augmentation de la synthèse protéique pourrait induire l'oncogenèse.

\section{De la signalisation à la traduction: PI3K/Akt/mTOR}

Les facteurs de croissance, les agents mitogènes et les hormones activent tous la voie PI3K/Akt/mTOR et la traduction cap-dépendante [8] (Figure 2). La PI3K (phosphatidyl-inositol-3-kinase) phosphoryle le phosphatidylinositol-4,5-bisphosphate (Ptdlns [4,5]P2) et produit le phosphatidylinositol-3,4,5-trisphosphate (Ptdlns[3,4,5]P3). Cette production de P3 entraîne l'activation de PDKl (3'-phosphoinositide dependent kinase 1), qui à son tour active Akt, une serine/thréonine kinase de la famille $C A M P$-dependent protein kinase $A$ / protein kinase $G /$ protein kinase $C(A G C)$. Cette activation est contrôlée par PTEN, une lipide-phosphatase qui déphosphoryle les P3 et les convertit en leur forme inactive P2. PTEN est également un gène suppresseur de tumeurs souvent muté dans nombre de cancers et de syndromes associés au cancer [9]. Le complexe TSC1/TSC2 possède une activité GTPasique inhibant Rheb, une GTPase de la famille de Ras. En phosphorylant TSC2, Akt inactive le complexe TSC1/TSC2 ce qui permet à Rheb d'activer mTOR (mammalian target of rapamycin), une serine/thréonine kinase de la famille des phosphatidylinositol kinase related protein kinase (PIKK) [10]), par un mécanisme encore indéterminé [9]. TSCl et TSC2 sont également des gènes suppresseurs de tumeurs souvent mutés dans la sclérose tubéreuse, une pathologie caractérisée par l'émergence d'hamartomes (tumeurs bénignes), principalement dans le tube digestif [9].

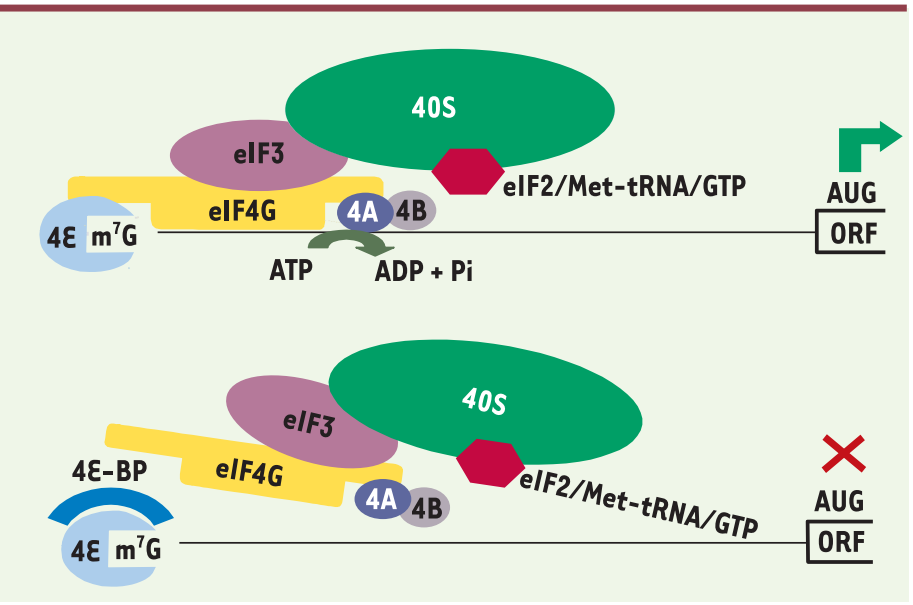

Figure 1. Contrôle de l'initiation de la traduction par 4E-BP. La fixation du complexe eIF4F (elF4E, elF4G et elF4A) à la coiffe de l'ARNm à traduire et à la sousunité ribosomique $40 S$ engendre un balayage du messager qui permet la localisation du codon d'initiation AUG. L'interaction de $4 \varepsilon-B P$ avec elF4E empêche l'interaction entre eIF4E et elF4G et, par conséquent, la formation du complexe elF4F. Pour leur part, elF4A et elF4B agissent, en tant que facteurs «accessoires », en dénaturant les structures secondaires de l'ARNm. Sous sa forme liée au GTP, elF2 interagit avec les nucléotides guanines et l'ARN de transfert méthionine (met-tARNi). Le complexe ternaire ainsi formé (elF2.GTP.met-tARNi) se lie à la sous unité ribosomique 40S. C'est alors l'anticodon de ce met-tARNi qui reconnaît le codon d'initiation présent dans l'ARNm. ORF : origin of replication.
Lorsque le ratio AMP/ATP est élevé, I'AMPK (kinase activée par l'AMP), senseur énergétique de la cellule, phosphoryle TSC2, ce qui augmente son activité et inhibe mTOR [9]. Enfin, les acides aminés et le glucose peuvent aussi activer directement mTOR [11].

Une fois activée, mTOR phosphoryle deux cibles majeures: les $S 6$ kinases (S6Kl et 2) et les 4E-BP (elF4E-binding proteins 1-3) [8] (Figure 2). L'hyperphosphorylation des $4 \varepsilon-B P$ (famille de trois protéines, 4E-BP1-3) diminue leur interaction avec elF4E et stimule la traduction cap-dépendante (Figure 2). Les 4E-BP sont aussi d'importants régulateurs de la croissance cellulaire car la surexpression d'un mutant constitutivement actif de $4 \varepsilon-B P 1$, où les quatre sites de phosphorylation (Thr36, Thr45, Ser64 et Thr 69) sont mutés en alanines, diminue la progression en phase $\mathrm{Gl}$ et bloque la transformation induite par c-Myc [12]. Jusqu'à récemment, on pensait que la $S 6$ kinase était absolument nécessaire à la traduction d'ARNm possédant une région riche en pyrimidines (TOP) dans leur partie non traduite (protéines ribosomiques efFl $\alpha$ ) [13]. Cette hypothèse a été réfutée en observant que chez les souris déficientes pour S6K1 et S6K2, la traduction des ARNm TOP est maintenue [14]. Cependant, la délétion de S6K1 et S6K2 mène à une réduction de la taille des souris, suggérant ainsi un nouveau rôle pour S6K dans la régulation de la croissance cellulaire [14].

mTOR permet l'intégration de nombreux signaux intra et extracellulaires et agit directement sur les cibles traductionnelles [8]. La rapamycine inhibe spécifiquement mTOR en se liant à son récepteur intracellulaire, la protéine FK506 binding protein-12 (FKBP12). Le complexe FKBP12/rapamycine ainsi formé inhibe l'activité kinase de mTOR [8]. Le développement d'analogues structuraux de la rapamycine, $\mathrm{CCl}-779$ (Wyeth-Ayerst), RAD001 (Novartis) et AP23573 (Ariad Pharmaceuticals) soulève un intérêt considérable. Ces analogues présentent, en effet, une puissante activité anti-proliférative dans plusieurs types de cancer: tumeurs pulmonaires, cérébrales, mammaires, coliques, leucémies et lymphomes. Plusieurs tests cliniques faisant appel à ces analogues sont actuellement en cours de validation [15, 16]. mTOR fait partie d'un large complexe protéique comprenant trois partenaires identifiés: raptor $[17,18], \mathrm{G} \beta \mathrm{L}$ [19] et rictor [20, 21] (Figure 2). Raptor (regulatory associated protein of mTOR, Kogl, chez la levure), qui interagit directement avec $4 \varepsilon-B P$ et $S 6 K$ grâce à leur motif TOS (séquence d'acides aminés: FEMDI et FDIDL, respectivement), est nécessaire à la phosphorylation de ces deux protéines par mTOR [22]. L'interaction raptor/mTOR est sensible à la disponibilité en nutriments et 
dépend d'un autre partenaire, G $\beta \mathrm{L}$ (Lst8p chez la levure) [19]. Indépendamment de raptor, $\mathrm{G} \beta \mathrm{L}$ interagit directement avec le domaine kinase de mTOR pour augmenter son activité. Enfin, rictor (rapamycin-insensitive companion of mTOR ou mAV03) intervient dans les réarrangements du cytosquelette $[20,21]$. Rictor et raptor délimitent deux complexes mTOR: alors que l'activité du complexe mTOR/G $\beta \mathrm{L} /$ raptor (mTORCl) est inhibée par la rapamycine, le complexe mTOR/rictor (mTORC2) y est insensible [20, 21] (Figure 2). De nouvelles données font intervenir le complexe mTOR/rictor dans la phosphorylation et l'activation d'Akt [23]. Ainsi, rictor pourrait être une cible thérapeutique dans le traitement des cancers associés à la voie PI3K/Akt/mTOR [23].

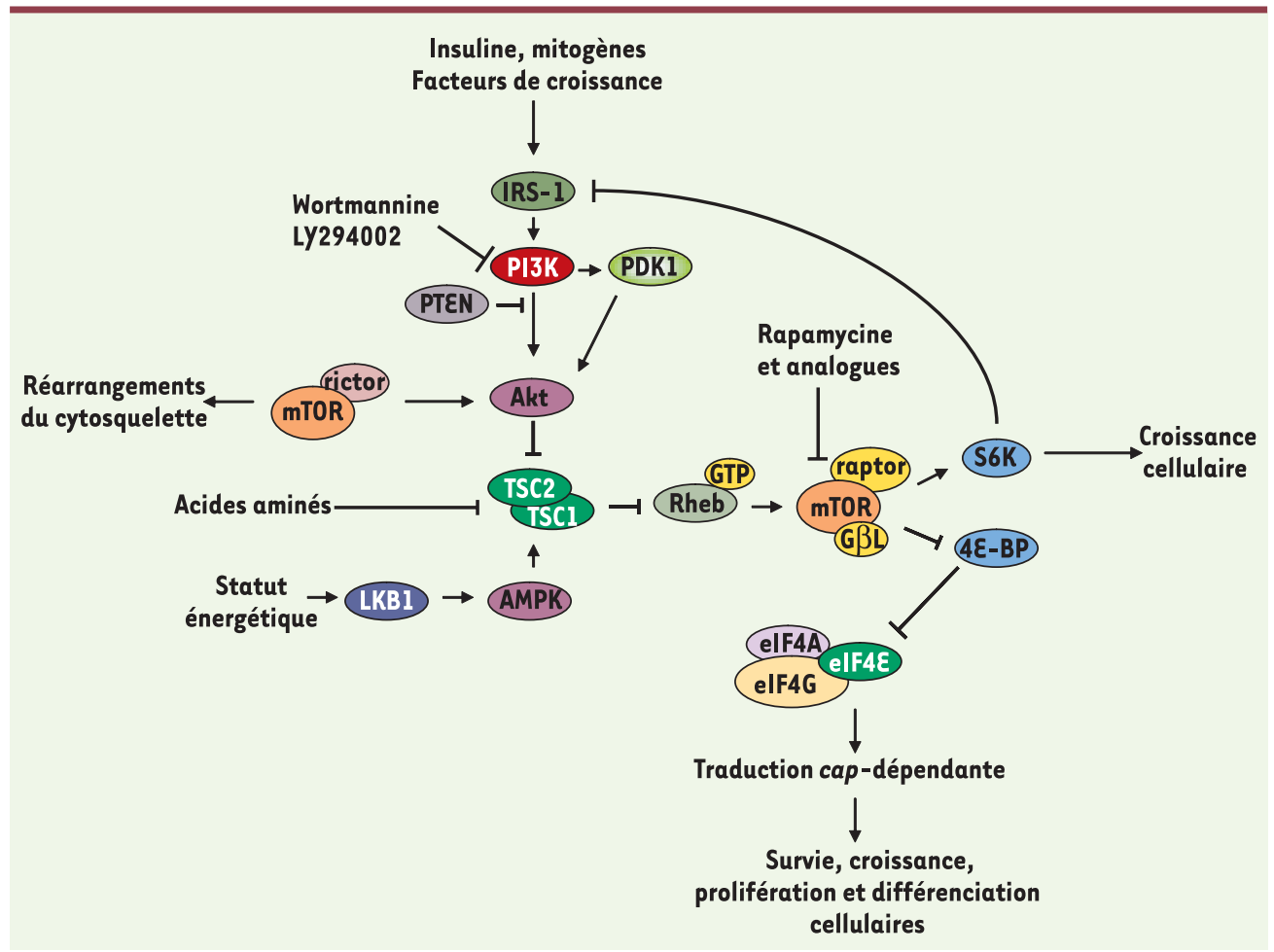

Figure 2. Intégration et régulation des signaux métaboliques par la voie PI3K/Akt/mTOR. La régulation de l'activité mTOR par les facteurs de croissance fait intervenir la voie PI3K/Akt. La PI3K phosphoryle le Ptdlns $(4,5)$ P2 et produit le PtdIns $(3,4,5) P 3$. La production de ce Ptdlns (3,4,5)P3 entraîne l'activation de PDKl qui, à son tour, active Akt. Cette activation est dépendante de PTEN. La worthmannine et le Ly294002 inhibent l'activité de la PI3K. Une fois activée, cette voie de signalisation provoque la phosphorylation et l'inhibition du complexe TSCl/ TSC2 par Akt, ce qui entraîne l'activation de Rheb (lié à GTP) et de mTOR. Le complexe TSC1/TSC2 joue un rôle central dans la régulation de l'activité mTOR: la présence d'acides aminés inhibe l'activité de ce complexe; à l'inverse, une carence énergétique provoque son activation via LKBl et AMPK. Une fois activé, le complexe mTOR/ raptor/Gß, sensible à la rapamycine, relaie ces signaux vers $4 \varepsilon-B P$ et $S 6 K$. La phosphorylation de $4 \varepsilon-B P$ permet la libération d'elF4E et la formation du complexe elF4F nécessaire à l'initiation de la traduction. De son côté, la phosphorylation de S6K entraîne un rétrocontrôle négatif de la voie PI3K/Akt empêchant ainsi toute suractivation de la traduction cap-dépendante. L'autre complexe mTOR/rictor, insensible à la rapamycine, joue un rôle dans le réarrangement du cytosquelette.

\section{Synthèse protéique, obésité et diabète}

\section{mTOR, adipogenèse et obésité}

La différenciation adipocytaire est un processus complexe, chronologiquement orchestré par les facteurs de transcription C/EBP et PPAR [24]. Dans un premier temps survient un arrêt de la prolifération des préadipocytes, puis C/EBP $\beta$ et $\delta$ sont exprimés de façon transitoire. le phénotype adipocytaire [24] (Figure 3).

Le rôle de mTOR dans l'adipogenèse a été mis en évidence par l'utilisation de la rapamycine. Cette dernière réduit la différenciation de préadipocytes humains [25]. L'effet de la rapamycine est indépendant de C/EBP $\beta$ et $C / \varepsilon B P \delta$, mais il est associé à une réduction de l'expression de $C / \varepsilon B P \alpha$ et PPAR $\gamma$. De plus, la rapamycine réduit l'activité transcriptionnelle de PPAR et perturbe la boucle de rétrocontrôle entre PPAR et $\mathrm{C} / \varepsilon \mathrm{BP} \alpha$ [26] (Figure 3), ce qui suggère une modulation transcriptionnelle plutôt que traductionnelle de l'adipogenèse par la rapamycine. Les rôles précis de $4 \varepsilon-B P I$ et S6K dans le processus d'adipogenèse sont encore mal connus. La rapamycine inhibe totalement la phosphorylation de S6K, mais partiellement la différenciation adipocytaire de cellules 3T3-L1 [27] et l'expression d'un mutant constitutivement actif de S6K n'est pas capable de rétablir ce processus [27]. À l'inverse, la rapamycine n'inhibe que partiellement la phosphorylation de $4 \varepsilon$ $B P 1$ [27], suggérant que $4 \varepsilon-B P 1$ pourrait jouer un rôle plus important que S6K dans la différenciation adipocytaire. II s'avère que les souris $4 \varepsilon-\mathrm{BPI}^{-/-}$présentent une masse adipeuse réduite du fait d'une dépense énergétique accrue [28], associée à une conversion du tissu adipeux blanc en tissu adipeux brun, détectée par l'expression d'UCP-1 (uncoupling protein-1). De 
plus, PGC-1 (PPAR $\gamma$ co-activator-1), facteur de transcription impliqué dans la biogenèse mitochondriale et la thermogenèse, est également surexprimé dans le tissu adipeux blanc des souris $4 \varepsilon-B P I^{-/-}$. L'ensemble de ces données suggère que le contrôle de l'adipogenèse par mTOR impliquerait elF4E/4E-BPl plutôt que S6K. Ces données accordent également un rôle complexe à la voie mTOR par laquelle l'activation de la voie elF4E/4EBPl ainsi que l'activité kinase de mTOR sont toutes deux nécessaires à l'initiation et au développement du processus adipogénique.

\section{mTOR et insulino-résistance}

Les conditions physiologiques favorisant une suractivation de la voie mTOR telles que l'hyperinsulinémie, l'excès chronique de nutriments et l'obésité, pourraient accroître l'insulino-résistance, cause de diverses maladies métaboliques, notamment le diabète de type 2 [29]. Une étude pionnière a montré qu'une exposition prolongée à l'insuline réduisait non seulement la mobilité éléctrophorétique, mais également le niveau protéique d'IRS-1 (insulin receptor substrate-1), protéine clé dans le relais des effets pléiotropiques de l'insuline [30]. Cette réduction démontrait des effets sur la transduction du signal puisque l'activation d'Akt, initialement stimulée, était réduite après

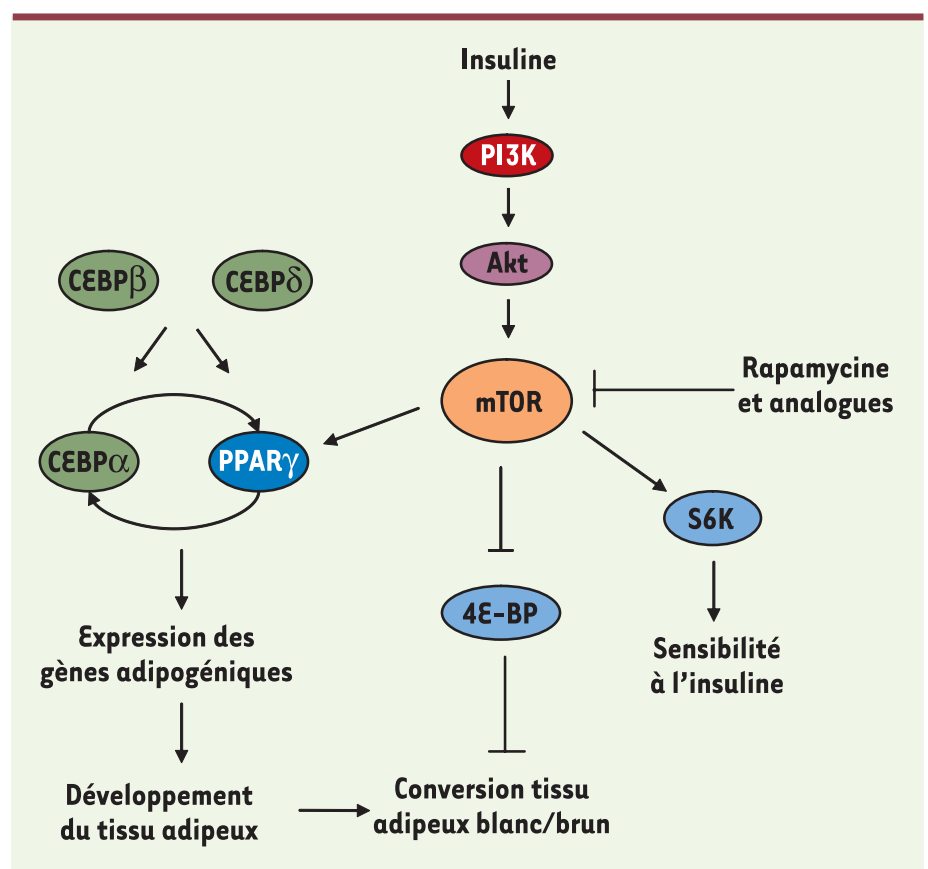

Figure 3. Régulation de l'adipogenèse et de la sensibilité à l'insuline par mTOR. La régulation transcriptionnelle de l'adipogenèse implique l'activation de plusieurs familles de facteurs de transcription. Ceux-ci sont chronologiquement exprimés : C/EBP $\beta$ et $\delta$, suivis de PPAR $\gamma$, qui à son tour active $C / \varepsilon B P \alpha$. $C / \varepsilon B P \alpha$, via un rétrocontôle positif sur PPAR $\gamma$, maintient la différenciation adipocytaire. L'adipogenèse fait également intervenir la voie mTOR. Une fois activée, elle contrôle l'expression et l'activité de PPAR $\gamma$, régulant de fait l'expression des gènes adipogéniques. Via $4 \varepsilon-B P$, mTOR module la différenciation fonctionnelle des tissus adipeux blanc et brun. Enfin, via la S6K, mTOR est également capable de réguler la sensibilité à l'insuline. une exposition prolongée à l'insuline. De plus, l'utilisation d'inhibiteurs de la voie PI3K/mTOR bloquait cet effet de l'insuline, tandis que l'utilisation d'une forme constitutivement active de la PI3K reproduisait les effets d'une exposition prolongée à l'insuline [30]. L'ensemble de ces données suggérait qu'un mécanisme mTOR-dépendant était la cause de l'inhibition de la voie PI3K/Akt via une boucle de rétrocontrôle négatif dont IRS-1 était la cible (Figure 2).

Récemment, le lien entre mTOR et le développement de I'insulino-résistance a été clarifié [29, 31, 32]. Les activités mTOR et S6K sont accrues dans le foie et le muscle squelettique de rats en régime gras [32]. Cette augmentation de l'activité mTOR est associée à une augmentation de la phosphorylation inhibitrice d'IRS-1 (Ser307, Ser636/639) et d'une inhibition d'Akt. À l'inverse, l'inhibition de mTOR permet de restaurer les activités PI3K et Akt [32]. Les délétions de TSCl ou TSC2 sont connues pour engendrer une suractivation de la voie mTOR et de S6K $[29,31]$. De façon similaire au régime gras, la suractivation de mTOR dans ces modèles réduisait l'activation d'Akt en réponse à l'insuline, à PDGF et au sérum [29, 31]. Cette inhibition de la voie Akt pouvait s'expliquer par une inhibition de l'expression d'IRS-1 [29]. II est ici remarquable que la délétion de S6KI corrige ce phénotype en restaurant l'activation d'Akt et l'expression d'IRS-1, suggérant que S6K1 maîtrise le rétrocontrôle négatif de la voie mTOR sur la voie PI3K/Akt [29] (Figure 2).

II restait à déterminer si une suractivation de la voie mTOR/ S6K pouvait contribuer au développement de l'insulinorésistance in vivo. L'étude de la souris $56 \mathrm{KI}^{-/-}$conforte l'idée selon laquelle S6K1 intervient dans l'inhibition d'IRS1 [33]. Les souris témoins sous régime gras présentaient une phosphorylation accrue de $\mathrm{S6Kl}$ et une réduction de la phosphorylation d'Akt dans le muscle, le foie et le tissu adipeux [33]. De plus, comparées aux souris $56 \mathrm{KI}^{-/-}$, les souris témoins obèses présentaient une augmentation de la phosphorylation d'IRS-1 sur les résidus Ser307 et Ser636/639 dans le tissu adipeux, sites associés à l'insulino-résistance [34]. De la même façon, la phosphorylation de ces sites et celle de $\mathrm{S} 6 \mathrm{Kl}$ étaient accrues chez des souris ob/ob et $K / K A^{y}$, deux modèles d'obésité génétique [33].

\section{Conclusions}

La traduction est une étape essentielle dans la régulation de l'expression de nombreuses protéines nécessaires à la croissance, la prolifération et la survie cellulaire. Les données discutées dans cette suite d'analyses tendent à démontrer qu'en plus de son rôle dans le développement tumoral, une suractivation de la voie mTOR semble également jouer un 
rôle prépondérant dans le développement du syndrome métabolique en régulant l'adipogenèse, ainsi que la sensibilité à l'insuline, via un rétrocontrôle négatif de S6K sur IRS-1. Dans un tel contexte, une meilleure compréhension du rôle respectif des protéines $4 \varepsilon$-BP et $S 6 K$ revêt une importance clinique majeure dans le traitement du cancer, de l'obésité, du diabète, ainsi que dans le développement de nouvelles stratégies thérapeutiques. La rapamycine est, entre autres, utilisée pour réduire les risques de rejets de greffes. Les tests cliniques sur la rapamycine pour le traitement des cancers sont également porteurs de grands espoirs. Enfin, le fait que la rapamycine in vitro soit capable d'inhiber la différenciation adipocytaire et d'accroître la sensibilité à l'insuline fait de cet inhibiteur un agent thérapeutique potentiel dans le traitement de ces maladies. $\diamond$

\section{REMERCIEMENTS}

Les auteurs tiennent à remercier le Dr Sonenberg pour son soutien et le Dr Pelletier pour la lecture critique du manuscrit. Olivier Le Bacquer bénéficie d'une bourse postdoctorale McGill Biological Chemistry Fellowship, Yvan Martineau d'une bourse postdoctorale de l'Association pour la Recherche sur le Cancer et Yaël Mamane d'une bourse postdoctorale de l'Institut National du Cancer du Canada.

\section{SUMMARY}

When translation arises from its TORpor

Gene regulation by transcriptional and post-translational mechanisms is implicated in the regulation of cellular homeostasis. Transcriptional deregulation has been largely documented in the etiology of diseases such as cancer, obesity and diabetes. During the past decade, the control of translation initiation by the PI3K/Akt/ mTOR pathway in the development of these pathologies has been documented. Rapamycin, a specific inhibitor of mTOR, demonstrates considerable anti-proliferative activity against numerous cancer types. Recent studies also demonstrated that rapamycin may be beneficial in the treatment of obesity and diabetes. Rapamycin and its analogs seem destined for a promising future and will help in the development of novel therapeutic strategies. $\diamond$

\section{RÉFÉRENCES}

1. Holland $\varepsilon C$, Sonenberg N, Pandolfi PP, Thomas G. Signaling control of mRNA translation in cancer pathogenesis. Oncogene $2004 ; 23: 3138-44$.

2. Gingras AC, Raught $B$, Sonenberg N. elF4 initiation factors: effectors of mRNA recruitment to ribosomes and regulators of translation. Annu Rev Biochem 1999; 68 : 913-63.

3. Mamane $Y$, Petroulakis $\varepsilon$, Rong $L$, et al. elF4E-from translation to transformation. Oncogene $2004 ; 23: 3172-9$.

4. Shi Y, Taylor SI, Tan SL, Sonenberg N. When translation meets metabolism: multiple links to diabetes. Endocrinol Rev $2003 ; 24: 91-101$.

5. Ruggero D, Montanaro L, Ma L, et al. The translation factor elF-4E promotes tumor formation and cooperates with c-Myc in lymphomagenesis. Nat Med $2004 ; 10: 484-6$.
6. Wendel HG, De Stanchina $\varepsilon$, Fridman JS, et al. Survival signalling by Akt and elF4E in oncogenesis and cancer therapy. Nature $2004 ; 428: 332-7$.

7. Avdulov S, Li S, Michalek V, et al. Activation of translation complex elF4F is essential for the genesis and maintenance of the malignant phenotype in human mammary epithelial cells. Cancer Cell $2004 ; 5: 553-63$.

8. Hay N, Sonenberg N. Upstream and downstream of mTOR. Genes Dev $2004 ; 18$ : 1926-45.

9. Inoki K, Corradetti MN, Guan KL. Dysregulation of the TSC-mTOR pathway in human disease. Nat Genet $2005 ; 37: 19-24$.

10. Richardson CJ, Schalm SS, Blenis J. PI3-kinase and TOR: PIKTORing cell growth. Semin Cell Dev Biol $2004 ; 15$ : 147-59.

11. Proud CG. mTOR-mediated regulation of translation factors by amino acids. Biochem Biophys Res Commun $2004 ; 313: 429-36$.

12. Lynch M, Fitzgerald C, Johnston KA, et al. Activated elF4E-binding protein slows Gl progression and blocks transformation by c-myc without inhibiting cell growth. J Biol Chem $2004 ; 279: 3327-39$.

13. Meyuhas 0 . Synthesis of the translational apparatus is regulated at the translational level. EurJ Biochem $2000 ; 267: 6321-30$.

14. Pende M, Um SH, Mieulet V. S6K1 $1^{(-/)} / \mathrm{S} 6 \mathrm{~K} 2^{(-/-)}$mice exhibit perinatal ethality and rapamycinsensitive 5'-terminal oligopyrimidine mRNA translation and reveal a mitogen-activated protein kinase-dependent S6 kinase pathway. Mol Cell Biol 2004 ; 24 : 3112-24.

15. Rao RD, Buckner JC, Sarkaria JN. Mammalian target of rapamycin (mTOR) inhibitors as anticancer agents. Curr Cancer Drug Targets 2004 ; 4:621-35.

16. Dutcher JP. Mammalian target of rapamycin (mTOR) inhibitors. Curr Oncol Rep 2004 ; $6: 111-5$.

17. Kim DH, Sarbassov DD, Ali SM, et al. mTOR interacts with raptor to form a nutrient-sensitive complex that signals to the cell growth machinery. Cell $2002 ; 110: 163-75$.

18. Hara K, Maruki $Y$, Long $X$, et al. Raptor, a binding partner of target of rapamycin (TOR), mediates TOR action. Cell $2002 ; 110: 177-89$.

19. Kim DH, Sarbassov DD, Ali SM, et al. GbetaL, a positive regulator of the rapamycin-sensitive pathway required for the nutrient-sensitive interaction between raptor and mTOR. Mol Cell $2003 ; 11: 895-904$

20. Sarbassov D, Ali SM, Kim DH, et al. Rictor, a novel binding partner of mTOR, defines a rapamycin-insensitive and raptor-independent pathway that regulates the cytoskeleton. Curr Biol $2004 ; 14$ : 1296-302.

21. Jacinto $\varepsilon$, Loewith $R$, Schmidt $A$, et al. Mammalian TOR complex 2 controls the actin cytoskeleton and is rapamycin insensitive. Nat Cell Biol $2004 ; 6: 1122-8$.

22. Yonezawa K, Tokunaga C, Oshiro N, Yoshino K. Raptor, a binding partner of target of rapamycin. Biochem Biophys Res Commun 2004 ; 313 : 437-41.

23. Sarbassov DD, Guertin DA, Ali SM, Sabatini DM. Phosphorylation and regulation of Akt/PKB by the rictor-mTOR complex. Science $2005 ; 307$ : 1098-101.

24. Rosen $\varepsilon D$, Walkey CJ, Puigserver P, Spiegelman BM. Transcriptional regulation of adipogenesis. Genes Dev $2000 ; 14$ : 1293-307.

25. Bell A, Grunder L, Sorisky A. Rapamycin inhibits human adipocyte differentiation in primary culture. Obes Res $2000 ; 8: 249-54$.

26. $\operatorname{Kim} J \varepsilon$, Chen J. Regulation of peroxisome proliferator-activated receptor-gamma activity by mammalian target of rapamycin and amino acids in adipogenesis. Diabetes 2004 ; $53: 2748-56$

27. El-Chaar D, Gagnon A, Sorisky A. Inhibition of insulin signaling and adipogenesis by rapamycin : effect on phosphorylation of p70 56 kinase versus elF4E-BP1. Int J Obes Relat Metab Disord $2004 ; 28: 191-8$.

28. Tsukiyama-Kohara K, Poulin F, Kohara $M$, et al. Adipose tissue reduction in mice lacking the translational inhibitor 4E-BP1. Nat Med $2001 ; 7: 1128-32$.

29. Harrington LS, Findlay GM, Lamb RF. Restraining PI3K: mTOR signalling goes back to the membrane. Trends Biochem Sci $2005 ; 30: 35-42$.

30. Haruta T, Uno T, Kawahara J, et al. A rapamycin-sensitive pathway down-regulates insulin signaling via phosphorylation and proteasomal degradation of insulin receptor substrate-1. Mol Endocrinol $2000 ; 14: 783-94$.

31. Zhang H, Cicchetti G, Onda H, et al. Loss of Tscl/Tsc2 activates mTOR and disrupts PI3K-Akt signaling through downregulation of PDGFR. J Clin Invest 2003 ; 112 : 1223-33.

32. Khamzina L, Veilleux A, Bergeron S, Marette A. Increased activation of the mammalian target of rapamycin pathway in liver and skeletal muscle of obese rats: possible involvement in obesity-linked insulin resistance. Endocrinology $2005 ; 146: 1473-81$.

33. Um SH, Frigerio F, Watanabe M, et al. Absence of $\mathrm{S} 6 \mathrm{Kl}$ protects against age- and diet-induced obesity while enhancing insulin sensitivity. Nature $2004 ; 431: 200-5$.

34. Bouzakri K, Roques M, Gual P, et al. Reduced activation of phosphatidylinositol-3 kinase and increased serine 636 phosphorylation of insulin receptor substrate- 1 in primary culture of skeletal muscle cells from patients with type 2 diabetes. Diabetes $2003 ; 52: 1319-25$.

\section{TIRÉS À PART}

0. Le Bacquer 